\title{
Intake of black-vinegar-mash-garlic enhances salivary release of secretory IgA: A randomized, double-blind, placebo-controlled, parallel-group study
}

\author{
YASUSHI NAKASONE ${ }^{1}$, NORIMASA SATO ${ }^{2}$, TAKAYUKI AZUMA ${ }^{3}$ and KEIJI HASUMI ${ }^{4}$ \\ ${ }^{1}$ Kenkoukazoku Co., Ltd., Kagoshima 892-0848; ${ }^{2}$ Umeda Oak Clinic, Osaka 530-0057; ${ }^{3}$ Nihonbashi Sakura Clinic, \\ Tokyo 103-0025; ${ }^{4}$ Department of Applied Biological Science, Tokyo Noko University, Tokyo 183-8509, Japan
}

Received February 11,2016; Accepted April 27, 2016

DOI: $10.3892 /$ br.2016.687

\begin{abstract}
Several previous studies have provided evidence that suggests the beneficial effects of garlic and black vinegar on human health, including benefits to immune function. The preliminary study indicated that the intake of blackvinegar-mash-garlic-containing food, created from aged garlic pickled in the mash of black vinegar, enhanced the release of secretory immunoglobulin A ( $\operatorname{SIgA}$ ) in the saliva. The aim of the present study was to evaluate the effect of the food in a randomized, double-blind, placebo-controlled, parallel-group trial. The trial was conducted in subjects aged between 30 and 60 years whose rate of salivary SIgA release was moderately low. Subjects consumed $2.49 \mathrm{~g}$ of placebo or black-vinegar-mash-garlic-containing food (active food) daily for 8 weeks. The data obtained with 54 eligible subjects ( $\mathrm{n}=28$ and 26 for placebo and active, respectively) were analyzed for efficacy. The rates of salivary sIgA release in the active food group $(35.9 \pm 84.6$ and $47.9 \pm 123.4 \mu \mathrm{g} / \mathrm{min}$ at weeks 4 and 8 of intake; changes from pretrial value) were higher compared to the respective rates in the placebo food group $(-12.3 \pm 72.1$ and $-3.2 \pm 85.9 \mu \mathrm{g} / \mathrm{min}, \mathrm{P}=0.028$ and 0.082 , respectively). These findings indicate that intake of black-vinegar-mash-garlic-containing food enhanced the intraoral immune response. There was no adverse event associated with the intake of active food.
\end{abstract}

\section{Introduction}

Garlic (Allium sativum) is a plant closely associated with onion, shallot and leek. For thousands of years, it has been used as a medicinal agent for prevention and treatment of diseases

Correspondence to: Mr. Yasushi Nakasone, Kenkoukazoku Co., Ltd., 10-2 Hirano-cho, Kagoshima 892-0848, Japan

E-mail: yasushi.nakasone@kenkoukazoku.co.jp

Key words: black vinegar, garlic, secretory immunoglobulin A, immune function, clinical study due to its potential effects, which include antimicrobial, antithrombotic, hypolipidemic, antiarthritic, hypoglycemic, immunomodulatory and antitumor activities (1-5). Kurozu is a type of traditional Japanese black vinegar made from unpolished rice. During its manufacturing processes, saccharification, ethanol fermentation and acetic fermentation occur in one pot. Due to its appearance, the vinegar is known as black vinegar. Previous studies have suggested the benefits of black vinegar, including antioxidant, hypotensive and antitumor effects (6-9). Black-vinegar-mash-garlic is a food produced by pickling garlic in black vinegar mash and allowing further fermentation thereafter. The extracts of the whole fermentation product are rich in amino acids, organic acids, vitamins and minerals, and are commercially distributed as a health supplement.

Diet can be one of the factors that modify immune responses (10). Our preliminary investigation suggested that the intake of black-vinegar-mash-garlic-containing food (the commercial brand 'Jukusei Kurozuninniku Premium') enhanced the immune function as represented by stimulation of secretory immunoglobulin A ( $\operatorname{Ig}$ A) release in the saliva (data unpublished). Salivary sIgA is synthesized as dimeric IgA by plasma cells in salivary glands and constitutes a section of the first line of defense against pathogen invasion (11). It can serve as a marker reflecting the immune function in the intraoral and upper respiratory tract (12). Increasing evidence suggests the potential use of salivary sIgA to predict mucosal and systemic immunity (13). In the present study, the effect of black-vinegar-mash-garlic-containing food on salivary sIgA release was investigated in a randomized, double-blind, placebo-controlled, parallel-group trial.

\section{Materials and methods}

Test articles. The test articles, placebo food and black-vinegar-mash-garlic-containing food (active food) were produced by Kenkoukazoku Co., Ltd. (Kagoshima, Japan). The ingredients of the test articles are shown in Table I. Subjects were administered 6 capsules (a total of $2.49 \mathrm{~g}$ ) of either the placebo or active food every day for 8 weeks during the test period. The dose of black-vinegar-mash-garlic-containing food was defined based on its recommended daily intake. 
Study design. A randomized, double-blind, placebo-controlled, parallel-group study was conducted at two centers, Yaesu Sakura-dori Clinic (presently Nihonbashi Sakura Clinic, Tokyo, Japan), and Umeda Oak Clinic (Osaka, Japan), under the supervision by the principle investigators Dr Takayuki Azuma (Yaesu Sakura-dori Clinic) and Dr Norimasa Sato (Umeda Oak Clinic). The study was conducted between May and October 2014. The study conformed to the principles of the Declaration of Helsinki and was approved on April 24, 2014 by the institutional review board of the incorporated medical institution of Aiseikai Aisei Hospital Ueno Clinic (Tokyo, Japan) on the basis of the protocol and information on the test study. The study was registered as the ID no. UMIN000013933 (Effects of food containing Kurozu Moromi and garlic powder on immune function: A randomized, double blind, placebo-controlled study) at the UMIN Clinical Trials Registry, Japan.

The details of this study were disclosed to subjects prior to the start of the study, and the investigators obtained informed consent from each subject. The candidates underwent pretrial testing (life style questionnaire, health check, somatometry, physical examination, fasting blood tests, urinalysis, salivary sIgA determination and T-cell subset analysis) and began documenting the pretrial lifestyle. As outlined in Fig. 1, 60 subjects (34 males and 26 females) were included out of 120 subjects who underwent pretrial testing. The major criteria for inclusion of subjects were those with a moderately slow rate of salivary $\operatorname{sIg} \mathrm{A}$ release and with their age between 30 and 60 years. Inclusion criteria for the study were: i) Males or females whose ages were between 30 and 60 years; ii) those with a moderate rate of salivary $\operatorname{sIg}$ A release. Exclusion criteria for the study were: i) Those who habitually consume foods rich in black vinegar or garlic; ii) those who habitually take health food or a supplement that may enhance immune function; iii) those with a case history of an allergic disease such as pollinosis, allergic rhinitis, asthma, atopic dermatitis and allergic conjunctivitis; iv) those who are receiving a treatment that will affect this study such as desensitization therapy; v) those who have been receiving within 1 month before the entry to this study or are planning to receive dental or intraoral treatment; vi) those who have dental or intraoral trouble; vii) those who have been engaged in shift work or heavy physical work; viii) those who habitually undergo hard exercise such as long distance running; ix) those who have difficulties undergoing testing in this study, such as sampling of saliva; $\mathrm{x})$ those who have suffered from any disease or who require any medication; xi) those who are suffering from or have a case history of disease such as diabetes mellitus, liver disease, kidney disease, heart disease, or disease that affects corticoid secretion; xii) those who may be liable to allergies in response to the test article; xiii) those who are judged inappropriate as participants from the pretrial test; xiv) those who are planning to get pregnant or nurse a baby during this study; $\mathrm{xv}$ ) those who have been participating in any other clinical trial within 1 month or who are planning to do such after agreeing informed consent of joining this study; xvi) those who are judged inappropriate as participants from the answers to the lifestyle questionnaire; xvii) those who are judged inappropriate as participants from the view of the principle investigators. The selected candidates were assigned to the placebo food group
Table I. Composition of the test foods.

\begin{tabular}{lrr}
\hline Composition & Placebo & Active \\
\hline Ingredient & & \\
Linseed oil, mg & $1,098.0$ & $1,035.0$ \\
Starch, mg & 430.8 & 430.8 \\
Acetic acid flavor, mg & 90.0 & 0.0 \\
Caramel pigment, mg & 30.0 & 0.0 \\
Black-vinegar-mash-garlic powder, mg & 0.0 & 300.0 \\
Glycerol, mg & 363.6 & 363.6 \\
Beeswax, mg & 252.0 & 195.0 \\
Gelling agent, mg & 165.6 & 165.6 \\
Nutritional content & & \\
Energy, kcal & 15.67 & 14.69 \\
Protein, g & 0.001 & 0.06 \\
Fat, g & 1.35 & 1.07 \\
Carbohydrate, g & 0.88 & 1.20 \\
Sodium, mg & 8.19 & 6.72 \\
\hline
\end{tabular}

Values are for the daily intake of the test food (6 capsules).

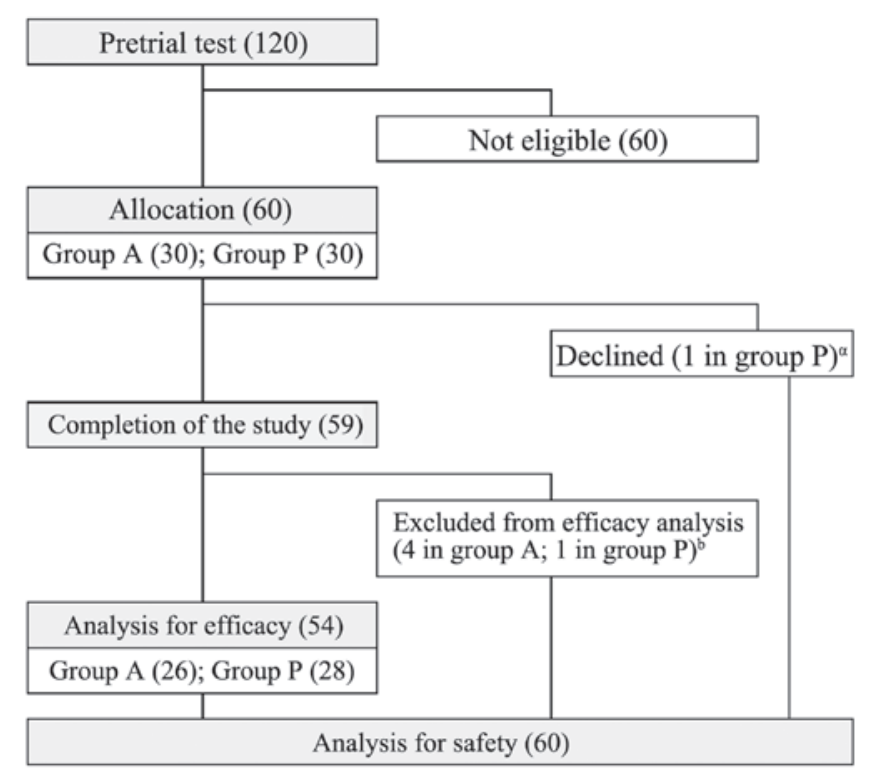

Figure 1. Flowchart of the study population selection. The figures in the parentheses denote the number of subjects. The inclusion of the subjects was judged by the principle investigators. In group A, subjects consumed black-vinegar-mash-garlic-containing food (active-food), and in group P,

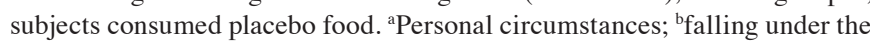
exclusion criteria for efficacy analysis.

$(n=30)$ or active food group $(n=30)$ based on random number tables. The allocation was performed by Mr. Yoshihisa Kibune (TTC Co., Ltd., Tokyo, Japan) and was concealed from the subjects, the investigators and the researchers who recruited and assessed the participating subjects. The participants ingested the placebo or active food every day for 8 weeks and noted the time and amount of their test article intake, as well as their use of pharmaceuticals. At weeks 4 and 8 of the study, participants underwent a health check, somatometry, physical 
Table II. Background characteristics of the subjects.

\begin{tabular}{lccc}
\hline Characteristics & $\begin{array}{c}\text { Active food } \\
\text { group }\end{array}$ & $\begin{array}{c}\text { Placebo food } \\
\text { group }\end{array}$ & P-value \\
\hline Subject number & $\begin{array}{c}26 \text { (male 11; } \\
\text { female 15) }\end{array}$ & $\begin{array}{c}28 \text { (male 19; } \\
\text { female 9) }\end{array}$ & 0.057 \\
& $44.5 \pm 7.9$ & $44.2 \pm 8.3$ & 0.871 \\
Age, year & $163.52 \pm 9.25$ & $167.25 \pm 7.44$ & 0.108 \\
Height, cm & $59.67 \pm 12.31$ & $63.89 \pm 10.48$ & 0.180 \\
Body weight, kg & $22.13 \pm 2.93$ & $22.77 \pm 2.96$ & 0.427 \\
Body mass & & & \\
index, kg/m ${ }^{2}$ & $173.46 \pm 58.22$ & $179.67 \pm 54.35$ & 0.686 \\
Rate of salivary & & & \\
sIgA release, $\mu$ g/min & $42.5 \pm 6.6$ & $45.0 \pm 7.7$ & 0.195 \\
CD4 $^{+}$T-cell, $\%$ & $28.1 \pm 6.3$ & $27.1 \pm 5.2$ & 0.537 \\
CD $^{+}$T-cell, $\%$ & $1.58 \pm 0.54$ & $1.71 \pm 0.57$ & 0.367 \\
CD4 $^{+} / \mathrm{CD}^{+}$ratio & & & \\
\hline
\end{tabular}

Values are the mean \pm standard deviation. $\operatorname{sIgA}$, secretory immunoglobulin $\mathrm{A} ; \mathrm{CD}$, cluster of differentiation.

Table III. Effect of the intake of black-vinegar-mash-garlic-containing food on the distribution of T-cell subset population.

Change from

pretrial value

T-cell subset

distribution

\begin{tabular}{llllc}
\hline $\mathrm{CD}^{+} \mathrm{T}$-cell, $\%$ & A & 26 & $-2.2 \pm 3.2$ & $-0.1 \pm 3.1$ \\
& $\mathrm{P}$ & 28 & $-3.1 \pm 4.0$ & $-0.2 \pm 3.9$ \\
$\mathrm{CD} 8^{+}$T-cell, $\%$ & A & 26 & $0.3 \pm 3.5$ & $0.5 \pm 3.3$ \\
& P & 28 & $-0.9 \pm 4.0$ & $-0.5 \pm 3.8$ \\
$\mathrm{CD}^{+} / \mathrm{CD}^{+}$ratio & A & 26 & $-0.09 \pm 0.28$ & $0.01 \pm 0.35$ \\
& P & 28 & $-0.04 \pm 0.28$ & $0.06 \pm 0.25$
\end{tabular}

Values are the mean \pm standard deviation. No significant differences were observed between groups at each occasion. $\mathrm{CD}$, cluster of differentiation; group A, subjects consumed black-vinegar-mash-garlic-containing food (active-food); group P, subjects consumed placebo food.

examination, salivary sIgA determination and T-cell subset analysis. At week 8, participants also underwent fasting blood tests and urinalysis.

During this trial, the principal investigators and their assistants directed the participants with respect to the following points: i) No change to lifestyle, including diet, alcohol consumption, exercise and sleeping; ii) avoid over-exercise, abstemious eating or overeating; iii) no change to exercise habits; iv) no consumption of health food, dietary supplement, lactic acid bacteria beverage, yogurt, kimchi or lactobacillus preparation; v) log the name and dose when a participant uses pharmaceuticals; vi) consume the specified amount of the test article and to log the time and amount of the

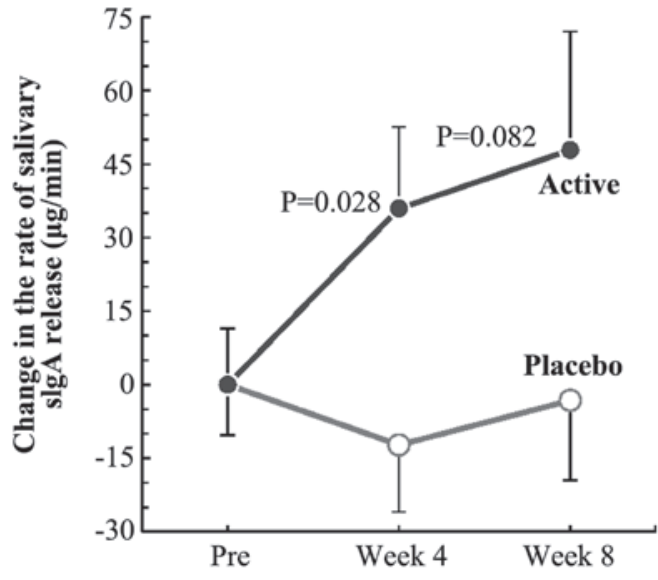

Figure 2. Time course of the effect of the intake of black-vinegar-mash-garlic-containing food on the rate of salivary sIgA release. The change in the rate of salivary sIgA release following the initiation of daily intake of test article, placebo food or black-vinegar-mash-garlic-containing food (active), is shown. Values obtained at weeks 4 and 8 for each subject were subtracted by the respective value in the pretrial test. Each value represents the mean \pm standard error of the mean $(n=28$ for placebo food group and 26 for active food group). sIgA, secretory immunoglobulin A.

intake every day; vii) avoid alcohol consumption and ingestion of pharmaceuticals on the day before testing, and avoid hard exercise on the day and the day before testing; ix) to finish eating a meal by 10:00 p.m. and thereafter not to take any meal other than warm water until the end of testing; $x$ ) to go to bed by 12:00 p.m. on the day before testing; xi) to brush teeth without using toothpaste on the day and the day before testing; and xii) to avoid smoking following toothbrushing until the end of the testing on that day.

The primary outcome measure was the rate of salivary sIgA release, and the secondary outcome measure was distribution of T-cell subset population [percentages of cluster of differentiation $4^{+}\left(\mathrm{CD}^{+}\right)$and $\mathrm{CD}^{+} \mathrm{T}$ cells in peripheral blood lymphocytes, as well as the ratio of $\mathrm{CD}^{+} \mathrm{T}$ cells to $\mathrm{CD} 8^{+}$ T cells].

Measurements of salivary sIgA. Subjects rinsed their mouth twice and gargled twice with $150 \mathrm{ml}$ of water for each time before testing. After sitting for $15 \mathrm{~min}$, subjects underwent sampling of their saliva using a Salisoft (Sarstedt, Numbrecht, Germany) for $120 \mathrm{sec}$. The amount of saliva recovered was recorded, and the salivary concentration of sIgA was determined by immunonephelometry according to the standard laboratory protocol of the BML Inc. (Tokyo, Japan).

T-cell subset analysis. The cell surface CD4 and CD8 antigens on peripheral blood lymphocytes were analyzed by flow cytometry using fluorescent-labeled monoclonal antibodies specific for the respective cell surface antigens (cat. nos. 340133 and 347313, respectively; BD Biosciences, Tokyo, Japan) according to the standard laboratory protocol of the LSI Medience Corporation (Tokyo, Japan).

Statistical analysis. All the measured values are expressed as the mean \pm standard error, or as indicated, the error bars may represent the standard error of the mean. For comparison of the values in the placebo and active food groups, $\chi^{2}$ test (for 
gender) or unpaired Student's t-test (for others) were employed, and $\mathrm{P}<0.05$ was considered to indicate a statistically significant difference.

\section{Results}

Subjects. In total, 60 subjects were enrolled and randomly allocated to each of the 2 groups: Group P in which participants received placebo food, or group A in which participants received black-vinegar-mash-garlic-containing food (Fig. 1). The subjects ingested 6 capsules of the respective test article every day. One participant in group P excluded themselves from the study at day 4 . The remaining 59 participants completed the study. However, 5 subjects were judged to fall into exclusion criteria and were eliminated from the efficacy analysis for the following reasons: Diagnosed as diabetes mellitus $(\mathrm{n}=1$ in group A), lifestyle change due to hospitalization for lumbar disc herniation ( $\mathrm{n}=1$ in group $\mathrm{A}$ ), and long-term cold that may affect sIg $\mathrm{A}$ release ( $\mathrm{n}=2$ in group $\mathrm{A}$ and $\mathrm{n}=1$ in group $\mathrm{P})$. Thus, the data obtained with 54 participants $(\mathrm{n}=26$ in group $\mathrm{A}$ and $\mathrm{n}=28$ in group $\mathrm{P}$ ) were used for the efficacy analysis (Fig. 1).

Background data. The background data of the participants who were subjected to efficacy analysis are shown in Table II. No significant difference was observed in the values between groups $\mathrm{A}$ and $\mathrm{P}$ for each item, including the rate of salivary sIgA release and T-cell subset population.

Effects of black-vinegar-mash-garlic-containing food intake. In group A, the rate of salivary $\operatorname{sg} \mathrm{A}$ release was significantly high $(\mathrm{P}=0.028)$ compared to that in group $\mathrm{P}$ at week 4 of the study (Fig. 2). The rate in group A at week 8 was also higher than that in group $P$. The time course of the changes from the pretrial values suggested a trend of elevation of the rate of SIgA secretion following the intake of active test article (blac k-vinegar-mash-garlic-containing food) (Fig. 2). No significant difference was observed between groups $\mathrm{A}$ and $\mathrm{P}$ in the population distribution of $\mathrm{CD} 4^{+}$and $\mathrm{CD} 8^{+}$peripheral lymphocytes following the test article consumption (Table III).

Adverse event. There was one serious adverse event, the herniation of the lumbar disc, with a subject in group A. Since its emergence was accidental associated with exercise, the principle investigator judged this case unrelated to the test article consumption. There were certain other non-serious adverse events during this study. Except for one observation in one subject in group P, which was a stomach ache that relieved following cessation of test article intake and was judged associated with the test article intake, the other events were judged by the principle investigators to be unrelated to test article consumption.

\section{Discussion}

The present study aimed to investigate the immunomodulatory effect of the intake of black-vinegar-mash-garlic-containing food, which has been commercially distributed as a health food since February 2012. Following this, <93 million capsules have been consumed. The results in the present study demonstrated that the subjects who ingested the black-vinegar-mash-garlic-containing food for 4 weeks had a significantly higher rate of salivary release of $\operatorname{sig} \mathrm{A}$ compared to those who consumed the placebo food. Thus, it is suggested that the intake of black-vinegar-mash-garliccontaining food is beneficial in enhancing intraoral mucosal immunity.

SIgA constitutes a major mucosal immune effector molecule and provides an important first line of defense against pathogens, such as Streptococcus mutans and Porphyromonas gingivalis $(14,15)$. Luminal SIgA is postulated to interfere with pathogen adherence to mucosal epithelial cells and promote intracellular neutralization of pathogens. IgA-secreting cells differentiate in lymphoid tissues associated with mucosa (15). The majority of the IgA-secreting cells reside in the gut mucosa. Increasing evidence suggests that the migration of antibody-secreting cells to the gut is important in conferring protective immune responses (16). The test food used in the present study was provided as a capsule, and the gastrointestinal tract was the first tissue to be exposed to the ingredients it contained. Thus, it is likely that the immune system in the gastrointestinal tract is first stimulated by the intake of black-vinegar-mash-garlic-containing food, and this may in turn affect salivary sIgA release through migration of the antibody-secreting cells to the intraoral mucosal tissues.

In conclusion, the present findings indicate that the intake of black-vinegar-mash-garlic-containing food stimulates intraoral immune function. There was no adverse event associated with the consumption of the food. Further investigations, including in vitro experiments and animal studies, may reveal the essential ingredient molecule and mechanism of its action involved in this immunomodulatory action.

\section{Acknowledgements}

YN is an employee of Kenkoukazoku Co., Ltd., which provided the research expenses to TTC Co., Ltd. YN was not involved in the interpretation of the results, and did not influence the outcomes at any stage of the clinical trial. A randomized, double-blind, placebo-controlled, parallel-group study was conducted with the aid of a fund from the Kenkoukazoku Co., Ltd.

\section{References}

1. Thomson M and Ali M: Garlic [Allium sativum]: A review of its potential use as an anti-cancer agent. Curr Cancer Drug Targets 3: 67-81, 2003.

2. Bayan L, Koulivand PH and Gorji A: Garlic: A review of potential therapeutic effects. Avicenna J Phytomed 4: 1-14, 2014.

3. Schäfer $\mathrm{G}$ and Kaschula $\mathrm{CH}$ : The immunomodulation and anti-inflammatory effects of garlic organosulfur compounds in cancer chemoprevention. Anticancer Agents Med Chem 14: 233-240, 2014.

4. Kyo E, Uda N, Kasuga S and Itakura Y: Immunomodulatory effects of aged garlic extract. J Nutr 131: 1075S-1079S, 2001.

5. Lamm DL and Riggs DR: Enhanced immunocompetence by garlic: Role in bladder cancer and other malignancies. J Nutr 131: 1067S-1070S, 2001.

6. Shimoji Y, Tamura Y, Nakamura Y, Nanda K, Nishidai S, Nishikawa $Y$, Ishihara $\mathrm{N}$, Uenakai $\mathrm{K}$ and Ohigashi H: Isolation and identification of DPPH radical scavenging compounds in Kurosu (Japanese unpolished rice vinegar). J Agric Food Chem 50: 6501-6503, 2002.

7. Nanda K, Miyoshi N, Nakamura Y, Shimoji Y, Tamura Y, Nishikawa Y, Uenakai K, Kohno H and Tanaka T: Extract of vinegar 'Kurosu' from unpolished rice inhibits the proliferation of human cancer cells. J Exp Clin Cancer Res 23: 69-75, 2004. 
8. Fukuyama N, Jujo S, Ito I, Shizuma T, Myojin K, Ishiwata K, Nagano M, Nakazawa $\mathrm{H}$ and Mori $\mathrm{H}$ : Kurozu moromimatsu inhibits tumor growth of Lovo cells in a mouse model in vivo. Nutrition 23: 81-86, 2007

9. Sugiyama S, Kishi M, Fushimi S, Oshima Y and Kajimoto O: Hypotensive effect and safety of brown rice vinegar with high concentration of GABA on mild hypertensive subjects. Jpn Pharmacol Ther 36: 429-444, 2008.

10. Lin WW and Karin M: A cytokine-mediated link between innate immunity, inflammation, and cancer. J Clin Invest 117 $1175-1183,2007$.

11. Woof JM and Mestecky J: Mucosal immunoglobulins. Immunol Rev 206: 64-82, 2005
12. Dodds MW, Johnson DA and Yeh CK: Health benefits of saliva: A review. J Dent 33: 223-233, 2005.

13. Brandtzaeg P: Do salivary antibodies reliably reflect both mucosal and systemic immunity? Ann N Y Acad Sci 1098: 288-311, 2007.

14. Russell MW, Hajishengallis G, Childers NK and Michalek SM: Secretory immunity in defense against cariogenic mutans streptococci. Caries Res 33: 4-15, 1999.

15. Woof JM and Kerr MA: The function of immunoglobulin A in immunity. J Pathol 208: 270-282, 2006.

16. Mora JR and von Andrian UH: Differentiation and homing of IgA-secreting cells. Mucosal Immunol 1: 96-109, 2008. 